\title{
Heart Failure Progression in Hypertrophic Cardiomyopathy - Possible Insights From Cardiopulmonary Exercise Testing -
}

\author{
Damiano Magrì, MD, PhD; Federica Re, MD; Giuseppe Limongelli, MD; \\ Piergiuseppe Agostoni, MD; Elisabetta Zachara, MD; Michele Correale, MD; \\ Vittoria Mastromarino, MD; Caterina Santolamazza, MD; Matteo Casenghi, MD; \\ Giuseppe Pacileo, MD; Fabio Valente, MD; Marco Morosin, MD; \\ Beatrice Musumeci, MD; Erika Pagannone, MD, PhD; Antonello Maruotti, PhD; \\ Massimo Uguccioni, MD; Massimo Volpe, MD; Camillo Autore, MD
}

\begin{abstract}
Background: Heart failure (HF) progression and its complications represent major emergent concerns in hypertrophic cardiomyopathy $(\mathrm{HCM})$. We investigated the possible adjunctive role of cardiopulmonary exercise testing (CPET) in predicting HF-related events. An exercise-derived risk model, the HYPertrophic Exercise-derived Risk HF (HYPERHF), has been developed.
\end{abstract}

\begin{abstract}
Methods and Results: A multicenter cohort of 620 consecutive HCM outpatients was recruited and followed (2007 to 2015). The endpoint was death from HF, cardiac transplantation, NYHA III-IV class progression, severe functional deterioration leading to hospitalization for septal reduction, and hospitalization for HF worsening. During a median follow-up of 3.8 years (25-75th centile: $2.3-5.3$ years), 84 patients reached the endpoint. Peak circulatory power (peak oxygen consumption * peak systolic blood pressure), ventilatory efficiency and left atrial diameter were independently associated with the endpoint and, accordingly, integrated into the HYPERHF model (C index: 0.849; best cutoff value equal to $15 \%$ ).
\end{abstract}

Conclusions: CPET is useful in the evaluation of HCM patients. In this context, the HYPERHF score might allow early identification of those patients at high risk of HF progression and its complications. (Circ J 2016; 80: 2204-2211)

Key Words: Cardiopulmonary exercise test; Heart failure; Hypertrophic cardiomyopathy; Prognosis

$\mathbf{H}$ ypertrophic cardiomyopathy (HCM), the most common inherited heart disease, is characterized by markedly different morphologic, functional, and clinical spectra. ${ }^{1}$ Besides the improvement in strategies for primary prevention of sudden cardiac death (SCD), ${ }^{2,3}$ recent major advances have been made in pharmacological and nonpharmacological approaches specifically directed to minimize/contain the overall cardiovascular mortality and morbidity. ${ }^{4-6}$ The direct consequence of such comprehensive management was that HCM evolved to a treatable disorder associated with a nearly normal life expectancy and that the greatest challenge now is to investigate possible strategies for early identification of those patients at high risk of cardiovascular events and, specifically, of heart failure (HF) development/progression. ${ }^{7}$ In this context, previous studies suggested the cardiopulmonary exercise testing (CPET) as a useful approach to objectively assessing functional capacity in HCM patients. ${ }^{8,9}$ Most recently, there are increasing reports about a possible role of CPET assessment in stratifying the overall HCM prognosis, ${ }^{10-12}$ even including SCD risk. ${ }^{13}$ Indeed, most of the HCM

Received May 8, 2016; revised manuscript received August 9, 2016; accepted August 16, 2016; released online September 13, 2016 Time for primary review: 37 days

Department of Clinical and Molecular Medicine, University of Rome "La Sapienza", Rome (D.M., V.M., C.S., M. Casenghi, B.M., E.P., M.V., C.A.); Cardiology Division, Cardiac Arrhythmia Center and Cardiomyopathies Unit, San Camillo-Forlanini Hospital, Rome (F.R., E.Z., M.U.); Cardiology SUN, Monaldi Hospital, II University of Naples, Naples (G.L., G.P., F.V.); "Monzino" Cardiologic Center, IRCCS, Milan (P.A., M.M.); Department of Clinical Sciences and Community Health, University of Milan, Milan (P.A.); Department of Cardiology, University of Foggia, Foggia (M. Correale); Cardiovascular Department, "Ospedali Riuniti" Trieste and Postgraduate School Cardiovascular Sciences, University of Trieste, Tieste (M.M.); Department of Economic, Political Sciences and Modern Languages "Libera Università-SS Maria Assunta", Rome (A.M.), Italy; Centre for Innovation and Leadership in Health Sciences, University of Southampton, Southampton (A.M.), UK; and IRCCS-Neuromed, Pozzilli (IS) (M.V.), Italy

Mailing address: Magrì Damiano, MD, PhD, Department of Clinical and Molecular Medicine, University of Rome "La Sapienza", Rome, Italy. Email: damiano.magri@uniroma1.it

ISSN-1346-9843 doi:10.1253/circj.CJ-16-0432

All rights are reserved to the Japanese Circulation Society. For permissions, please e-mail: cj@j-circ.or.jp 
pathophysiological features, closely implicated in reducing exercise capacity, influence the progression to $\mathrm{HF}$ as well as the arrhythmic propensity in HCM patients. ${ }^{8,9,13-15}$

The current multicenter prospective study investigated a possible adjunctive role of CPET-derived parameters over the main clinical variables in predicting HF-related events in a large contemporary HCM cohort. We also sought to derive a possible exercise-derived risk model, namely the HYPertrophic Exercise-derived Risk HF (HYPERHF) score, that can be used to generate individualized risk estimates for HF-related events.

\section{Methods}

\section{Study Sample}

A total of 683 consecutive outpatients with HCM were recruited and prospectively followed in 5 HCM Italian centers between September 2007 and October 2015: Azienda Ospedaliera Sant'Andrea, "Sapienza" University, Rome $(n=379)$; Azienda Ospedaliera San Camillo Forlanini, Rome $(n=221)$; Ospedale Monaldi-Second University of Naples, Naples $(n=52)$; Centro Cardiologico Monzino, University of Milan, Milan ( $n=20)$; Ospedali Riuniti, University of Foggia, Foggia $(n=11)$. The diagnosis of HCM was based on a maximal wall thickness (MWT) $\geq 15 \mathrm{~mm}$ unexplained by abnormal loading conditions or in accordance with published criteria for the diagnosis of disease in relatives of patients with unequivocal disease., ${ }^{4,5}$ Patients with known metabolic diseases or syndromic causes of HCM were excluded from the present study.

The study complied with the ethical standards of the Declaration of Helsinki and was reviewed and approved by the institutional ethics committees. Written informed consent was given by all participants. The authors from each participating center guarantee the integrity of the data from their institution and have agreed to the article as written.

\section{Patients Clinical Assessment}

Data were independently collected at each participating center using a uniform methodology. Each HCM patient who fulfilled the initial inclusion criteria underwent a de novo clinical assessment, including anamnesis with pedigree analysis and New York Heart Association (NYHA) classification, 24-h ECG Holter monitoring, transthoracic Doppler echocardiography and maximal CPET. The usual 5 SCD risk factors were also collected: (a) FH-SCD (history of HCM-related SCD in at least 1 first-degree or other relatives $<50$ years old); (b) massive LV hypertrophy (MWT $\geq 30 \mathrm{~mm}$ ); (c) at least 1 run of nonsustained ventricular tachycardia (NSVT) ( $\geq 3$ consecutive ventricular beats at $\geq 120$ beats/min and $<30$ s in duration on $24-h$ ECG Holter monitoring); (d) unexplained syncope judged inconsistent with neurocardiogenic origin; (e) abnormal blood pressure response to exercise (ABPRE) (failure to increase systolic blood pressure (SBP) by at least $20 \mathrm{mmHg}$ from rest to peak exercise or a fall of $\geq 20 \mathrm{mmHg}$ from SBP). ${ }^{4-6,16}$

The following echocardiographic measurements, obtained according to international guidelines, ${ }^{17}$ were considered: LV end-diastolic diameter (LVEDd, parasternal long axis), the greatest LV thickness (MWT, measured at any LV site), left atrial diameter (LAd, parasternal long axis), the highest maximal LV outflow tract gradient among those measured at rest, in the orthostatic position and after the Valsalva maneuver (LVOTGmax, apical 4-chamber view), ${ }^{18}$ and LV ejection fraction with Simpson's biplane methods (LVEF, apical 4-chamber view).

All CPET was performed using an electronically braked cycle-ergometer. A personalized ramp exercise protocol was performed, aiming for a test duration of $10 \pm 2 \mathrm{~min} .{ }^{19}$ The exercise was preceded by few minutes of resting breath-by-breath gas exchange monitoring and by a 3-min unloaded warm-up. In the absence of clinical events, CPET was interrupted when patients stated that they had reached maximal effort. A 12-lead ECG, DBP and SBP were recorded during CPET, in order to obtain the following parameters: resting heart rate (HR), peak HR, \%pHR ([peak HR/(220-age)] $* 100),{ }^{20}$ and $\Delta$ SBP (peak SBP-rest SBP). A breath-by-breath analysis of expiratory gases and ventilation (VE) was performed, and peak values were obtained in the last 20 s of exercise. The predicted peak $\mathrm{VO}_{2}$ was determined by using the sex-, age-, and weight-adjusted formulas. ${ }^{21}$ Circulatory power $\left(\mathrm{CP}=\right.$ peak $\left.\mathrm{VO}_{2} * \mathrm{SBP}\right)$ was obtained considering peak $\dot{\mathrm{VO}}_{2}$ value both as $\mathrm{ml} / \mathrm{kg}$ and as a percentage of predicted $(\mathrm{CP} \%) .{ }^{22}$ The $\dot{\mathrm{VO}}_{2} /$ workload relationship was measured throughout the entire exercise. Anaerobic threshold (AT) was measured by $\mathrm{V}$-slope analysis of $\dot{\mathrm{VO}}_{2}$ and $\dot{\mathrm{VCO}}_{2}$, and was confirmed by ventilator equivalents and end-tidal pressures of $\mathrm{CO}_{2}$ and $\mathrm{O}_{2}$. The end of the isocapnic buffering period was identified when $\dot{\mathrm{VE}} / \dot{\mathrm{VCO}}_{2}$ increased and the end-tidal pressure of $\mathrm{CO}_{2}$ decreased. The $\dot{\mathrm{VE}} / \dot{\mathrm{V} C O} 2$ slope was calculated as the slope of the linear relationship between $\dot{\mathrm{VE}}$ and $\dot{\mathrm{VCO}}_{2}$ from the first minute after the beginning of the loaded exercise and the end of the isocapnic buffering period. ${ }^{21}$

\section{Clinical Outcomes}

All patients had planned clinical reviews every 6-12 months or earlier according to their clinical status. Follow-up duration was defined as the time interval between the CPET examination and either the first event or the last visit/telephone interview in the case of no events. The HF endpoint was represented by the following events: death from HF, cardiac transplantation, progression to NYHA class III-IV caused by endstage phase with or without LVEF $<50 \%$ (hypokinetic dilated phase or restrictive phenotype evolution), ${ }^{5}$ severe functional deterioration leading to hospitalization for septal reduction and hospitalization because of HF symptoms or signs development. Death from either ischemic or hemorrhagic stroke $(n=3)$ and nonfatal cerebrovascular events $(n=7)$, as well as $\operatorname{SCD}(n=4)$, aborted SCD $(n=3)$ and ICD appropriate interventions $(n=18)$, were excluded from the present analysis. The causes of death, as well as the other events, were ascertained by experienced cardiologists at each center using hospital and primary healthcare records, death certificates, postmortem reports, and interviews with relatives and/or physicians.

\section{Statistical Analysis}

Statistical analysis was performed using R (R Development Core Team, 2014). The survival package was used. A P-value $\leq 0.05$ was generally considered as statistically significant. As a preliminary analysis, an extension of the Shapiro-Wilk test of normality was performed. In comparing the 2 populations, the variance was estimated separately for both groups and the Welch-Satterthwaite modification to the degrees of freedom was used. Unless otherwise indicated, all data are expressed as mean \pm SD. Categorical variables were compared with a difference between proportion tests; a 2-sample t-test was used to compare the continuous data between the group with the endpoint and the one without. We focused on the distribution of the survival times by adopting the Cox proportional hazards regression model. We performed a backward selection of the predictors to be included in the model. A 5\% significance level was used in the backward elimination procedure to select covariates for the final multivariate model. To avoid 


\begin{tabular}{|c|c|c|c|c|}
\hline & Overall & HF e & point & P yalu \\
\hline & Sample $(n=620)$ & Yes $(n=84)$ & No $(n=536)$ & r value \\
\hline \multicolumn{5}{|l|}{ General data } \\
\hline Age, years & $49 \pm 16$ & $48 \pm 18$ & $49 \pm 16$ & 0.6 \\
\hline Male, n (\%) & $425(69)$ & $43(51)$ & $382(71)$ & $<0.001$ \\
\hline Age at diagnosis, years & $38.7 \pm 17.8$ & $35 \pm 19$ & $39 \pm 18$ & NS \\
\hline NYHA III, n (\%) & $35(5.7)$ & $13(15.5)$ & $22(4.1)$ & 0.04 \\
\hline LVOTO, n (\%) & $200(32)$ & $35(42)$ & $165(30)$ & 0.05 \\
\hline Myectomy, n (\%) & $39(6.3)$ & $5(5.9)$ & $34(6.3)$ & NS \\
\hline ICD, n (\%) & $73(12)$ & $24(28)$ & $49(9)$ & $<0.001$ \\
\hline \multicolumn{5}{|l|}{ SCD risk factors } \\
\hline NSVT, n (\%) & $214(34.5)$ & $35(42)$ & 179 (33) & NS \\
\hline FH-SCD, n (\%) & $71(11.5)$ & $16(19)$ & $55(10)$ & 0.02 \\
\hline MWT >30 mm, n (\%) & $42(6.8)$ & $11(13)$ & $31(6)$ & 0.01 \\
\hline Syncope, n (\%) & $94(15.2)$ & $22(26)$ & $72(13)$ & 0.002 \\
\hline ABPRE, n (\%) & $112(18.1)$ & $37(44)$ & $75(14)$ & $<0.001$ \\
\hline \multicolumn{5}{|l|}{ Echocardiography } \\
\hline LVEDd, mm & $46.4 \pm 5.1$ & $47.4 \pm 7.2$ & $46.2 \pm 4.6$ & NS \\
\hline LAd, mm & $42.3 \pm 7.4$ & $46.1 \pm 7.3$ & $41.8 \pm 7.2$ & $<0.001$ \\
\hline MWT, mm & $20.4 \pm 4.8$ & $21.3 \pm 5.3$ & $20.2 \pm 4.7$ & NS \\
\hline LVOTGmax, mmHg & $26 \pm 29$ & $33.1 \pm 36$ & $24.8 \pm 27$ & $<0.001$ \\
\hline LVEF, \% & $62 \pm 7$ & $57 \pm 9$ & $63 \pm 6$ & $<0.001$ \\
\hline$\Delta \mathrm{SBP}, \mathrm{mmHg}$ & $44 \pm 24$ & $25 \pm 20$ & $46 \pm 24$ & $<0.001$ \\
\hline Peak HR, \% & $81 \pm 14$ & $74 \pm 15$ & $82 \pm 13$ & $<0.001$ \\
\hline AT $\dot{\mathrm{VO}}_{2}, \mathrm{ml} / \mathrm{kg}$ & $14.4 \pm 4.2$ & $11.6 \pm 3.3$ & $14.6 \pm 4.2$ & $<0.001$ \\
\hline Peak $\dot{V O}_{2}, \mathrm{ml} / \mathrm{kg} / \mathrm{min}$ & $21.1 \pm 6.8$ & $15 \pm 4.9$ & $22 \pm 6.6$ & $<0.001$ \\
\hline Peak $\dot{V O}_{2}, \%$ & $71 \pm 19$ & $54 \pm 16$ & $74 \pm 19$ & $<0.001$ \\
\hline $\mathrm{CP}, \mathrm{ml} / \mathrm{kg}^{*} \mathrm{mmHg}$ & $3.525 \pm 1.442$ & $2.178 \pm 912$ & $3.735 \pm 1.396$ & $<0.001$ \\
\hline $\mathrm{CP} \%, \%{ }^{*} \mathrm{mmHg}$ & $11.934 \pm 4.426$ & $7.737 \pm 3.174$ & $12.591 \pm 4.232$ & $<0.001$ \\
\hline$\dot{\mathrm{VO}}_{2} / \mathrm{Wr}$ slope, $\mathrm{ml} /$ Watts & $10.5 \pm 1.9$ & $9.2 \pm 2.1$ & $10.8 \pm 1.7$ & $<0.001$ \\
\hline$\dot{\mathrm{VE}} / \dot{\mathrm{VCO}}_{2}$ slope & $28.7 \pm 5.8$ & $34.7 \pm 7.2$ & $27.7 \pm 4.9$ & $<0.001$ \\
\hline \multicolumn{5}{|l|}{ Medications } \\
\hline$\beta$-blocker, n (\%) & $418(67)$ & $62(74)$ & $356(66)$ & NS \\
\hline Verapamil, n (\%) & $52(8)$ & $9(11)$ & $43(8)$ & NS \\
\hline Disopyramide, n (\%) & $27(4)$ & $6(7)$ & $21(4)$ & NS \\
\hline Amiodarone, n (\%) & $59(10)$ & $14(17)$ & $45(8)$ & 0.02 \\
\hline ACEI/ARB, n (\%) & $165(27)$ & $36(43)$ & $129(24)$ & $<0.001$ \\
\hline Diuretics, n (\%) & $157(25)$ & $36(43)$ & $121(23)$ & $<0.001$ \\
\hline
\end{tabular}

Data are expressed as mean $\pm \mathrm{SD}$, as absolute number of patients (\% on total sample). ABPRE, abnormal blood pressure response at exercise; ACEI/ARB, angiotensin-converting enzyme inhibitor/angiotensin-receptor blocker; AT, anaerobic threshold; $\mathrm{CP}$, circulatory power; $\mathrm{FH}$, family history; HR, heart rate; ICD, implantable cardioverter defibrillator; LAd, left atrial diameter; LVEDd, left ventricular end-diastolic diameter; LVEF, LV ejection fraction; LVOTGmax, maximal LV outflow tract gradient; MR, mitral regurgitation; MWT, maximum wall thickness; NSVT, nonsustained ventricular tachycardia; NYHA, New York Heart Association; $\Delta$ SBP, difference between peak and resting systolic blood pressure; SCD, sudden cardiac death; $\mathrm{V} E / \mathrm{V} \mathrm{CO}_{2}$ slope, relation between ventilation vs. carbon dioxide production; $\dot{\mathrm{VO}}_{2}$, oxygen consumption; $\dot{\mathrm{VO}}_{2} / \mathrm{Wr}$ slope, relation between $\dot{\mathrm{VO}}_{2}$ vs. work load.

the inclusion of collinear variables in the multivariate Cox analysis, we built several models in which $\dot{\mathrm{VO}}_{2}$-derived variables, as well as NYHA classification, known to be collinear, were added to the prognostic model one at a time. Conversely, to avoid an endogeneity issue, we avoided including in the multivariate model those variables pertaining to treatment at the study run-in (ICD presence and pharmacological treatment; see Table 1). We retained the model with the best trade-off between model complexity and model fit judged by the loglikelihood (number of variables included into the best model equal to 9, see Table 2). The risk model presented here was developed using the entire cohort ( $n=620$ patients). Discrimination of variables included in the final multivariate model specification was performed by Harrell's C index. Therefore, we investigated the proportional hazards assumption by tests and graphical diagnostics based on scaled Schoenfeld residuals. Tests of the proportional hazards assumption for each covariate were obtained by correlating the corresponding set of scaled Schoenfeld residuals with the Kaplan-Meier estimate of survival distribution. To check for the presence of influential observations, we produced a matrix of estimated changes in the regression coefficients after deleting each observation 


\begin{tabular}{|c|c|c|c|c|c|c|}
\hline \multirow{2}{*}{ Variables } & \multicolumn{3}{|c|}{ Univariate analysis } & \multicolumn{3}{|c|}{ Multivariate analysis } \\
\hline & Hazard ratio $(95 \% \mathrm{Cl})$ & $P$ value & C index & Hazard ratio $(95 \% \mathrm{Cl})$ & $P$ value & C index \\
\hline Male* $^{*}$ & $0.443(0.288-0.682)$ & 0.0002 & 0.592 & & & \multirow{16}{*}{0.849} \\
\hline NYHA III & $5.983(4.112-13.453)$ & 0.0001 & 0.641 & & & \\
\hline FH-SCD* & $1.919(1.112-3.313)$ & 0.0191 & 0.538 & & & \\
\hline Syncope* & $2.068(1.27-3.368)$ & 0.0030 & 0.535 & & & \\
\hline ABPRE & $4.49(2.91-6.93)$ & 0.0001 & 0.657 & & & \\
\hline $\mathrm{LAd}, \mathrm{mm}^{*}$ & $1.081(1.052-1.111)$ & 0.0001 & 0.686 & $1.0457(1.0154-1.0769)$ & 0.003 & \\
\hline LVEF$^{*}$ & $0.908(0.886-0.93)$ & 0.0001 & 0.666 & & & \\
\hline$\Delta \mathrm{SBP}$ & $0.96(0.95-0.97)$ & 0.0001 & 0.751 & & & \\
\hline Peak HR, \%* & $0.96(0.944-0.976)$ & 0.0001 & 0.643 & & & \\
\hline AT $\dot{\mathrm{VO}}_{2}, \mathrm{ml} / \mathrm{kg}$ & $0.81(0.743-0.882)$ & 0.0001 & 0.714 & & & \\
\hline Peak $\dot{V O}_{2}, \mathrm{ml} / \mathrm{kg} / \mathrm{min}$ & $0.801(0.76-0.843)$ & 0.0001 & 0.785 & & & \\
\hline Peak $\mathrm{VO}_{2}, \%$ & $0.935(0.921-0.949)$ & 0.0001 & 0.780 & & & \\
\hline $\mathrm{CP}, \mathrm{ml} / \mathrm{kg}^{*} \mathrm{mmHg}$ & $0.9986(0.9983-0.9989)$ & 0.0001 & 0.820 & & & \\
\hline $\mathrm{CP} \%, \%^{*} \mathrm{mmHg}^{*}$ & $0.9996(0.9996-0.9997)$ & 0.0001 & 0.813 & $0.9997(0.9996-0.9998)$ & 0.0001 & \\
\hline$\dot{\mathrm{VO}}_{2} / \mathrm{Wr}$ slope, $\mathrm{ml} / \mathrm{Watts}^{*}$ & $0.659(0.585-0.743)$ & 0.0001 & 0.681 & & & \\
\hline$\dot{\mathrm{VE}} / \dot{\mathrm{VCO}} 2$ slope $^{*}$ & $1.123(1.099-1.148)$ & 0.0001 & 0.792 & $1.074(1.044-1.104)$ & 0.0001 & \\
\hline
\end{tabular}

*Variables included in the best final multivariate model (adjusted for collinearity). $\mathrm{Cl}$, confidence interval. Other abbreviations as in Table 1.

in turn and comparing the magnitudes of the largest values to the regression coefficients. At last, a possible incorrect specification of the parametric form of the model was investigated. To detect nonlinearity, we constructed plots of the martingale residuals against covariates. For any 2 randomly chosen observations, agreement means that the observation with the shorter survival time of the 2 also has the larger risk score.

The probability of any HF-related events at 5 years for a single patient (ie, HYPertrophic Exercise-derived Risk score for HF-related events, HYPERHF) can be calculated using the following equation, derived from the Cox proportional:

HYPERHF: 1-So( $t)^{\exp (\text { Prognostic Index) }}$

where $\mathrm{S} 0(\mathrm{t})$ is the average survival probability at time $\mathrm{t}$ (ie, at 5 years), and the prognostic index is the sum of the products of the predictors and their coefficients estimated via the Cox model.

Finally, a receiver-operating characteristic (ROC) analysis was considered to determine the predictive capability of the

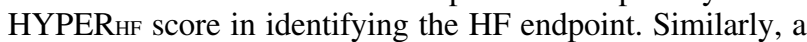
ROC analysis was also considered for each of the multivariate analyses resulting in continuous variables. The behavior of a cutoff-dependent performance measure, such as accuracy, was considered across the range of all cutoffs. Cutoff values were identified according to their accuracy [(true positive+true negative)/total sample] highest value. The optimal cutoff was the threshold that maximized the distance to the identity (diagonal) line according to Youden's J statistic.

\section{Results}

From the initial sample of 683 consecutive HCM outpatients, $63(9 \%)$ were excluded because metabolic maximal effort was not achieved (respiratory exchange ratio, peak $\dot{\mathrm{V} C O} /$ peak $\left.\dot{\mathrm{VO}}_{2}<1.05\right)$ or because the CPET data were not fully interpretable (ie, poor quality). A total of 620 patients were therefore considered for the present study.

\section{General Characteristics of the Study Population}

All the demographic and clinical characteristics of the entire cohort at study run-in are reported in Table 1 . The population mainly consisted of middle-aged male patients in NYHA I-II functional class $(94 \%)$ with none in NYHA IV; 32 patients $(5 \%)$ had atrial fibrillation, $39(6 \%)$ had echocardiographic evidence of endstage phase, $39(6 \%)$ had been myectomized and $73(12 \%)$ had been implanted with an ICD. Documented cardiovascular comorbidities included systemic hypertension $(26 \%)$, coronary artery disease $(6 \%)$ and diabetes $(5 \%)$. Pharmacologic treatment comprised $\beta$-blocker $(67 \%)$, verapamil $(8 \%)$, disopyramide $(4 \%)$, amiodarone $(10 \%)$, angiotensinconverting enzyme inhibitors or angiotensin-receptor blockers (27\%), and diuretics (25\%). During the entire follow-up, 28 (4\%) patients underwent myectomy because of worsening of HF symptoms/signs and $63(10 \%)$ additional patients underwent ICD implantation.

\section{Endpoint Analysis}

Median follow-up was 3.8 years (25-75th centile: $2.3-5.3$ years) with a total of 2,467 patient-years. During the entire followup, $84(13.5 \%)$ patients experienced at least one of the prespecified events. In patients who developed multiple events, time to the first event was used as the event time cutoff and, accordingly, HF-related events at 5-year cumulative hazard equal to 0.094 (95\% confidence interval, 0.056-0.132) was estimated. A total of $84 \mathrm{HF}$-related events were collected: HFrelated death occurred in 4 patients, 5 patients underwent heart transplantation, 24 patients evolved to an established NYHA III-IV class (16 patients due to hypokinetic phase and 8 patients due to restrictive phenotype evolution), 28 patients were hospitalized for septal reduction procedure for significant $\mathrm{HF}$ signs/symptoms development and, finally, 35 patients were hospitalized because of HF signs/symptoms. Patients who ended the follow-up period before the 5th year were considered censored at the time of the last clinical evaluation.

\section{Predictors of Outcome}

Table 1 shows the clinical characteristics of the patients 

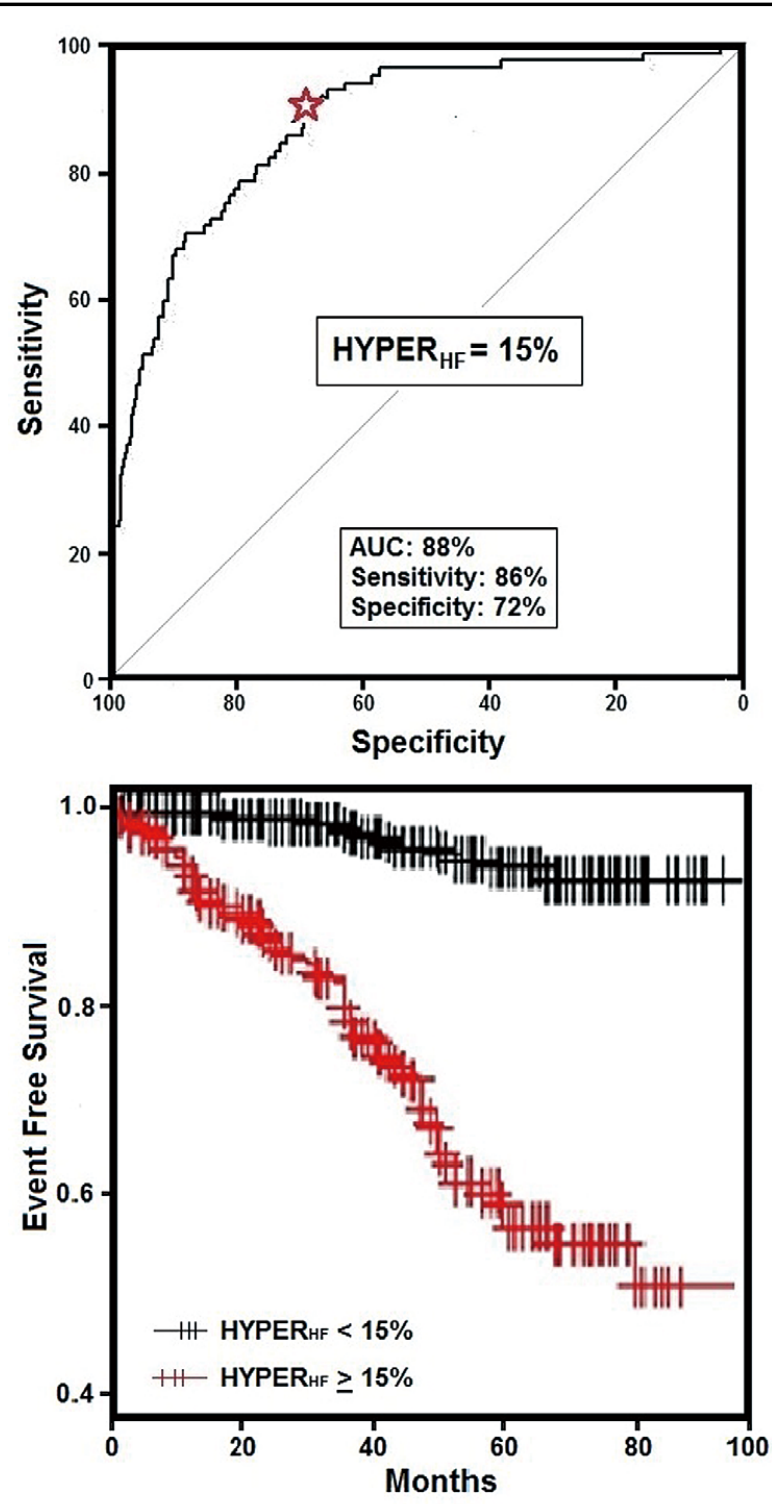

\begin{tabular}{|l|c|c|c|c|c|}
\cline { 2 - 6 } \multicolumn{1}{c|}{ HYPER $_{\text {HF }}$} & $\mathbf{1}^{\text {st }}$ year & $2^{\text {nd }}$ year & $3^{\text {rd }}$ year & $4^{\text {th }}$ vear & $5^{\text {th }}$ year \\
\hline$<\mathbf{1 5 \%}$ & 481 & 439 & 361 & 268 & 186 \\
\hline$\geq \mathbf{1 5 \%}$ & 139 & 113 & 88 & 69 & 47 \\
\hline
\end{tabular}

Figure 1. (Upper) Receiver-operating characteristic (ROC) analysis showing the point with the best sensitivity and specificity of the HYPertophic Exercise-derived Risk score (HYPERHF) value in the entire study sample $(n=620)$. (Lower) KaplanMeier estimator of HF-related events for the HYPERHF score according to a $15 \%$ cutoff value. See Table 2 for the original multivariate Cox proportional survival model and Table 3 for the accuracy data. HF, heart failure.

grouped according the study endpoint. Table 2 reports the detailed Cox proportional univariate and multivariate survival analyses for the HF endpoint. Covariates showing significant effects for the $\mathrm{HF}$ endpoint were: $\mathrm{CP} \%, \dot{\mathrm{VE}} / \dot{\mathrm{VCO}}_{2}$ slope and LAd.

\section{Model Presentation}

Given the Cox proportional multivariate survival analysis for the endpoint (Table 2), the probability of any HF-related events at 5 years for a single patient (ie, HYPertrophic Exercisederived Risk score for HF-related events, HYPERHF) can be calculated as:

$$
\hat{P}_{H F e v e n t s-a t-5 \text { years }}=1-0.910^{\exp (\text { Index })}
$$

where 0.910 is the survival probability at 5 years and index is the sum of the products of the (centered and scaled) covariates and their coefficients estimated via the Cox model [Index $=0.045 *$ LAd $(\mathrm{mm})-0.000285 * \mathrm{pVO}_{2} \mathrm{CP}$ (\% of predicted) $+0.071 * \dot{\mathrm{VE}} / \mathrm{V}^{\circ} \mathrm{O}_{2}$ slope $)$.

To avoid overflow in the argument to the exponential function, we scaled and centered the data. These actions did not change the result, but led to more numerical stability. An Excel Calculator has been supplied in Data S1.

According to the selected model, Harrell's C index was 0.8. A sensitivity analysis of model assumptions (validation) was also conducted: proportional hazards could not be rejected; atypical data did not influence the results and considering linear relationships in the model specification results was appropriate.

At ROC analysis the best HYPER $\mathrm{HF}_{\mathrm{H}}$ score cutoff value in predicting the primary endpoint was equal to $15 \%$ (area under the curve (AUC): $88 \%$ ) while the same analysis executed on each single continuous variable included in the abovementioned model showed the following best cutoff values: $\mathrm{CP}$

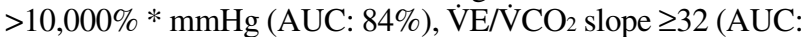
$81 \%$ ), LAd $>42 \mathrm{~mm}$ (AUC: $66 \%$ ) (Figures 1,2). A full description of the accuracy data for the HYPERHF score (3 possible cutoff values ranging from $10 \%$ to $20 \%$ ), as well as those for each single variable, is presented in Table 3.

\section{Discussion}

The present multicenter prospective study, conducted on wellcharacterized consecutive HCM outpatients regularly followed at 5 Italian HCM centers, shows that the $\mathrm{CP}$, the $\dot{\mathrm{VE}} / \mathrm{VCO}_{2}$ slope and the LAd were independently associated with the risk of HF-related events. Thus 2 out of the 3 abovementioned variables are obtainable uniquely from CPET analysis derived from a CPET assessment. The HYPERHF score represents the first attempt of an integrated risk prediction model potentially expandable to generating individualized risk estimates for HFrelated events in a contemporary HCM population.

Albeit SCD risk stratification in HCM patients still remains a challenging topic, ${ }^{2,3,13}$ it is undeniable that the epidemiology of HCM-related morbidity and mortality has evolved during the past decades, being HF development and its complications as major emergent concerns of the HCM spectrum. ${ }^{1,7,23}$ Supporting this changing scenario, in the present HCM population a total of 136 patients experienced at least one of the prespecified HF-related events, whereas SCD and its equivalents accounted for a total of 25 events. Accordingly, there is growing interest in investigating CPET analysis as an adjunctive tool to early identification of those patients at high risk of HF development/progression. ${ }^{9,24,25}$ Indeed, most of the pathophysiological features of HCM (ie, diastolic dysfunction, magnitude of LV hypertrophy, LVOT obstruction, myocardial ischemia and fibrosis, etc) are factors affecting both exercise capacity and prognosis. ${ }^{1,9,14,15}$ Most recently, 3 retrospective studies showed similar relationships between some CPET-derived variables and HCM prognosis. ${ }^{10-12}$ Specifically, in a large cohort of 1,005 consecutive HCM patients enrolled between 1997 and 2012 

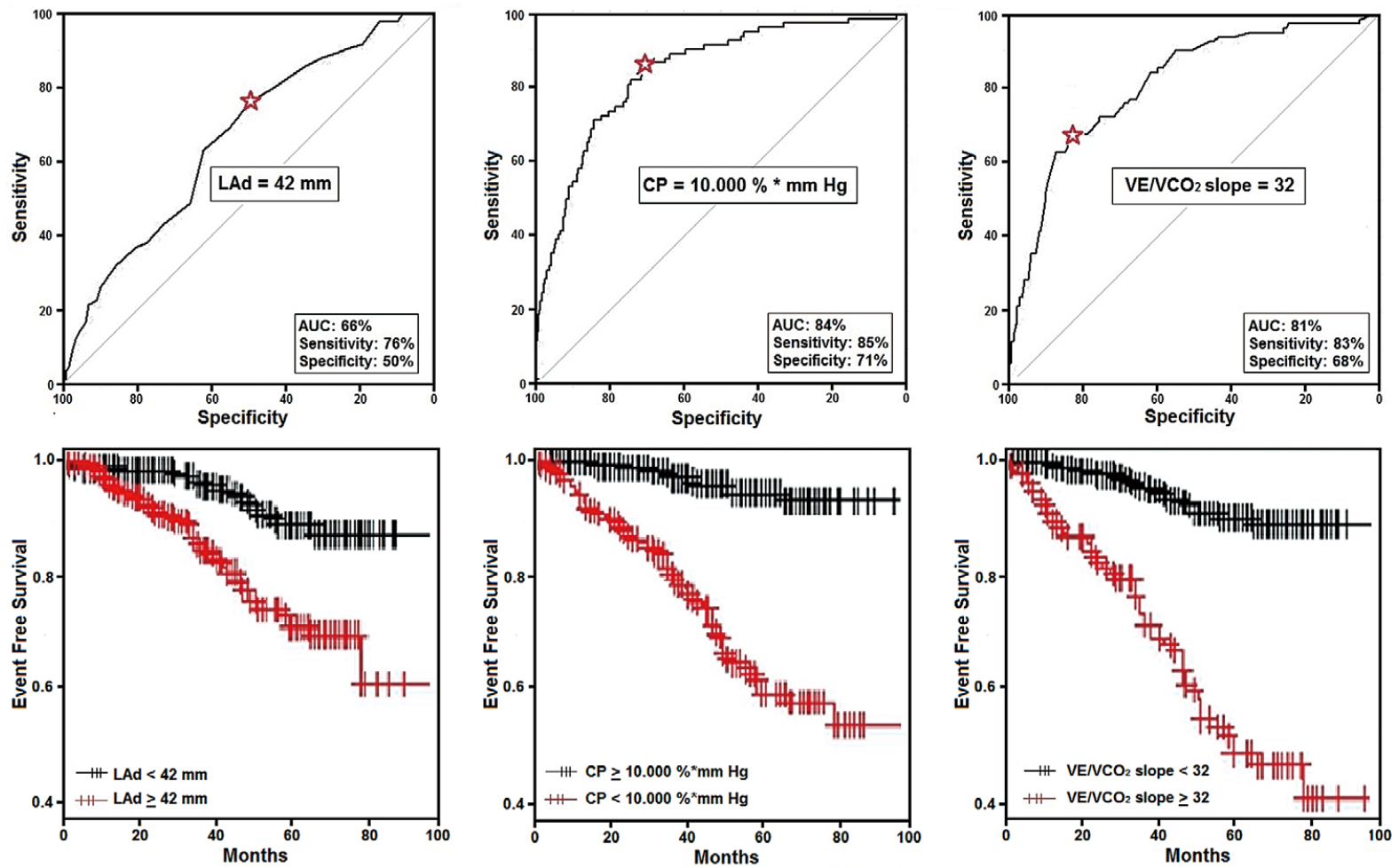

Figure 2. (Upper) Receiver-operating characteristic (ROC) analysis showing the point with the best sensitivity and specificity of each single variable included in the HYPERHF score. (Lower) Kaplan-Meier estimator of HF-related events according to the best cutoff values of each single variable included in the HYPERHF score. CP\%, circulatory power; HF, heart failure; VE/VCO 2 slope, ventilatory efficiency; Lad, left atrial diameter. See Table 2 for the original multivariate Cox proportional survival model and Table 3 for the accuracy data.

\begin{tabular}{|c|c|c|c|c|c|c|c|c|}
\hline & \multicolumn{4}{|c|}{ HF composite endpoint } & \multirow{3}{*}{ Sensitivity } & \multirow{3}{*}{ Specificity } & \multirow{3}{*}{ PPV } & \multirow{3}{*}{ NPV } \\
\hline & \multicolumn{2}{|c|}{ Yes $(n=84)$} & \multicolumn{2}{|c|}{ No $(n=536)$} & & & & \\
\hline & $\begin{array}{c}\text { True } \\
\text { positive }\end{array}$ & $\begin{array}{c}\text { False } \\
\text { negative }\end{array}$ & $\begin{array}{c}\text { False } \\
\text { positive }\end{array}$ & $\begin{array}{c}\text { True } \\
\text { negative }\end{array}$ & & & & \\
\hline \multicolumn{9}{|l|}{ HYPERHF score } \\
\hline$>10 \%$ & 79 & 5 & 214 & 322 & 0.94 & 0.60 & 0.27 & 0.98 \\
\hline$>15 \% *$ & $71^{*}$ & $13^{*}$ & $149^{*}$ & $387^{*}$ & $0.86^{*}$ & $0.72^{*}$ & $0.32^{*}$ & $0.97^{*}$ \\
\hline$>20 \%$ & 66 & 18 & 110 & 426 & 0.79 & 0.79 & 0.38 & 0.96 \\
\hline \multicolumn{9}{|l|}{ HYPERHF score variables } \\
\hline $\operatorname{LAd}(>42 \mathrm{~mm})$ & 64 & 20 & 270 & 266 & 0.76 & 0.50 & 0.19 & 0.93 \\
\hline Peak $\mathrm{VO}_{2} \mathrm{CP}(<10,000)$ & 71 & 13 & 154 & 382 & 0.84 & 0.71 & 0.31 & 0.97 \\
\hline$\dot{\mathrm{V}} \mathrm{E} / \mathrm{V} \mathrm{CO}_{2}$ slope $(>32)$ & 57 & 27 & 93 & 443 & 0.68 & 0.83 & 0.38 & 0.94 \\
\hline
\end{tabular}

${ }^{*}$ The HYPERHF score cutoff values with the best accuracy in predicting the HF endpoint. HYPERHF, Hypertrophic Exercise-derived Risk score for Heart Failure; PPV, positive predictive value; NPV, negative predictive value. Other abbreviations as in Table 1.

(5.5 years median follow-up, 63 events), Masri and colleagues showed that a reduced $\mathrm{p} \dot{\mathrm{VO}}_{2}(<50 \%$ of predicted) was independently associated with a composite outcome including overall mortality and SCD equivalent events. ${ }^{10}$ Similarly, in a much larger cohort of 1,898 patients enrolled between 1998 and 2010 (5.6 years median follow-up, 53 events), Coats and colleagues identified an independent relationship between $\mathrm{HF}$ death/heart transplantation and both $\mathrm{pVO}_{2}(\mathrm{ml} / \mathrm{kg} / \mathrm{min})$ and $\dot{\mathrm{VE}} / \dot{\mathrm{V} C O}{ }_{2}$ slope values. ${ }^{11}$ Lastly, although in a smaller but very well characterized cohort of $156 \mathrm{HCM}$ patients (2.1 years median follow-up, 21 events), Finocchiaro and colleagues also confirmed an independent role of $\mathrm{pVO}_{2}(<80 \%$ of predicted) and the $\dot{\mathrm{VE}} / \mathrm{V} \mathrm{CO}_{2}$ slope $(>34)$ in predicting a composite endpoint including overall mortality, heart transplantation and 
functional deterioration leading to hospitalization for septal reduction. ${ }^{12}$ In such a context, the present study not only strengthens the importance of exercise testing with gas-exchange analysis in HCM patients but adds some new data to the field. Indeed, besides its prospective design, the study analyzed a large contemporary HCM cohort on stable treatment (enrollment period between 2007 and 2015; 3.8 years median followup; 84 events) and it supplies for the first time an attempt at devising an integrated HF-related events risk prediction model derived from a large number of CPET-derived parameters on top of the main clinical variables, including those classically associated with HF development and/or progression in HCM. Moreover, in addition to LA enlargement, closely dependent on LV diastolic dysfunction, ${ }^{14,26}$ our multivariate risk prediction model included the $\dot{\mathrm{VE}} / \mathrm{V} \mathrm{CO}_{2}$ slope and, for the first time in this setting of patients, the circulatory power $\left[\mathrm{CP} \%=\mathrm{p} \dot{\mathrm{VO}}_{2}\right.$ (\% of maximum predicted) * peak SBP $(\mathrm{mmHg})]$. The latter CPET-derived parameter, according to its formula, ${ }^{22}$ magnifies the clinical and prognostic power of the ABPRE and the $\mathrm{p}^{\circ} \mathrm{O}_{2}$. Accordingly, the first variable depends on the intrinsic myocardial function/geometry as well on abnormal peripheral autonomic reflexes, ${ }^{27,28}$ whereas the second is a composite variable extensively shown to be a strong predictor of poor outcome in HF patients with systolic dysfunction. ${ }^{29,30}$ Most recently, in a relative large study sample, we showed a strong association between a reduced $\mathrm{p} \dot{\mathrm{VO}}_{2}$ and well-known markers of HCM disease severity and prognosis (ie, LAd, LVOT obstruction, $\triangle \mathrm{SBP}$, late gadolinium enhancement, etc). ${ }^{9}$ Similar results have been obtained in other studies dealing with this topic. ${ }^{8,14,15}$ Conversely, the $\dot{\mathrm{VE}} / \dot{\mathrm{VCO}}_{2}$ slope, besides its established independent prognostic role in HF with systolic dysfunction, has been suggested as a promising prognostic marker in HF with preserved LVEF., ${ }^{22,31-33}$ Indeed, ventilatory efficiency showed the best diagnostic accuracy for directly measured pulmonary capillary wedge pressure and LV diastolic properties at tissue Doppler analysis in not only these patients but also HCM patients. ${ }^{32,34}$ Furthermore, the $\dot{\mathrm{VE}} / \dot{\mathrm{VCO}}_{2}$ slope could unmask exercise-induced LV diastolic function derangement, given that the ventilation/perfusion mismatch phenomena caused by pulmonary arterial constriction increases in parallel with the exercise-related LV filling pressure increase. ${ }^{34}$

We also derived a cutoff value for each of the variables selected by the multivariate analysis, as well as for the HYPER score, thus supplying 2 possible, distinct approaches to HFrelated events risk management in HCM. However, albeit easy to use in daily clinical practice, each single cutoff has several limits to its clinical application. Accordingly, it seems to be more appropriate from the pathophysiological and clinical viewpoints to consider the HYPERHF score as a continuous variable.

Lastly, albeit not emerging from the multivariate analysis, our data highlighted a more likely progression towards $\mathrm{HF}$ development/progression in women. This finding is consistent with a previous report dealing with sex-related differences in HCM outcome, ${ }^{35}$ as well as with the recent paper by Coats et al in which female sex emerged as an independent predictor of overall cardiovascular mortality. ${ }^{11}$

\section{Study Limitations}

The relatively small number of patients enrolled, together with the low number of hard events, represents an obvious limitation that only allows us to suggest rather than affirm that the HYPERHF risk prediction model can really improve contemporary HCM clinical management. However, it should be noted that many other clinical and instrumental variables might be taken into account, as well as that no statistical model will be ever able to substitute for careful clinical assessment by a highly experienced physician. The present model particularly did not account for data on cardiac magnetic resonance imaging or for the possible effect of different gene mutations, both features shown to be closely related to HCM prognosis. ${ }^{36-39}$ Furthermore, we did not measure any circulating biomarkers (ie, natriuretic peptides, cardiac enzymes, etc), so we cannot support a recent interesting study showing that high CK-MB levels might predict HF progression in HCM patients. ${ }^{40}$ Finally, we examined the prognostic effect of several variables at a single time point; thus, we cannot exclude that changes in some variables, as for instance an upgrading of treatment during follow-up or upcoming risk factors, altered our survival analysis.

\section{Conclusions}

Our findings strengthen the emerging literature identifying exercise testing with gas-exchange analysis as a mandatory step in HCM clinical management, particularly in view of the growing incidence of $\mathrm{HF}$ progression and its complications. Only 3 variables, mostly exclusively CPET-derived, were independently predictive of HF development/worsening in our HCM cohort. In this context, the HYPERHF score represents the first attempt of an integrated risk prediction model finalized to help the physician in early identification of those HCM patients at high risk of HF progression. Further studies in larger HCM cohorts are needed to confirm the HYPERHF score's clinical value in routine HCM clinical management.

\section{Disclosures}

None to be declared.

\section{Funding}

The present work was partially supported by a research grant from "Sapienza" University of Rome to D.M.

\section{References}

1. Maron BJ, Maron MS. Hypertrophic cardiomyopathy. Lancet 2013; 381: $242-255$

2. Maron BJ. Risk stratification and role of implantable defibrillators for prevention of sudden death in patients with hypertrophic cardiomyopathy. Circ J 2010; 74: 2271 -2282.

3. O'Mahony C, Jichi F, Pavlou M, Monserrat L, Anastasakis A, Rapezzi C, et al; Hypertrophic Cardiomyopathy Outcomes Investigators. A novel clinical risk prediction model for sudden cardiac death in hypertrophic cardiomyopathy (HCM Risk-SCD). Eur Heart J 2014; 35: 2010-2020.

4. Gersh BJ, Maron BJ, Bonow RO, Dearani JA, Fifer MA, Link MS, et al. 2011 ACCF/AHA Guideline for the diagnosis and treatment of hypertrophic cardiomyopathy: Executive summary: A report of the American College of Cardiology Foundation/American Heart Association Task Force on practice guidelines. Circulation 2011; 124: 2761-2796.

5. Authors/Task Force members, Elliott PM, Anastasakis A, Borger MA, Borggrefe M, Cecchi F, Charron P, et al. 2014 ESC Guidelines on diagnosis and management of hypertrophic cardiomyopathy: The Task Force for the Diagnosis and Management of Hypertrophic Cardiomyopathy of the European Society of Cardiology (ESC). Eur Heart J 2014; 35: $2733-2779$.

6. JCS Joint Working Group. Guidelines for diagnosis and treatment of patients with hypertrophic cardiomyopathy (JCS 2012): Digest version. Circ J 2016; 80: 753-774.

7. Maron BJ, Ommen SR, Semsarian C, Spirito P, Olivotto I, Maron MS. Hypertrophic cardiomyopathy: Present and future, with translation into contemporary cardiovascular medicine. J Am Coll Cardiol 2014; 64: 83-99.

8. Sharma S, Elliott P, Whyte G, Jones S, Mahon N, Whipp B, et al. Utility of cardiopulmonary exercise in the assessment of clinical determinants of functional capacity in hypertrophic cardiomyopathy. Am J Cardiol 2000; 86: 162-168. 
9. Magrì D, Agostoni P, Cauti FM, Musumeci B, Egidy Assenza G, De Cecco $\mathrm{CN}$, et al. Determinants of peak oxygen uptake in patients with hypertrophic cardiomyopathy: A single-center study. Intern Emerg Med 2014; 9: 293-302.

10. Masri A, Pierson LM, Smedira NG, Agarwal S, Lytle BW, Naji P, et al. Predictors of long-term outcomes in patients with hypertrophic cardiomyopathy undergoing cardiopulmonary stress testing and echocardiography. Am Heart J 2015; 169: 684-692.

11. Coats CJ, Rantell K, Bartnik A, Patel A, Mist B, McKenna WJ, et al. Cardiopulmonary exercise testing and prognosis in hypertrophic cardiomyopathy. Circ Heart Fail 2015; 8: 1022-1031.

12. Finocchiaro G, Haddad F, Knowles JW, Caleshu C, Pavlovic A, Homburger J, et al. Cardiopulmonary responses and prognosis in hypertrophic cardiomyopathy: A potential role for comprehensive noninvasive hemodynamic assessment. JACC Heart Fail 2015; 3: $408-418$.

13. Magrì D, Limongelli G, Re F, Agostoni P, Zachara E, Correale M, et al. Cardiopulmonary exercise test and sudden cardiac death risk in hypertrophic cardiomyopathy. Heart 2016; 102: 602-609.

14. Sachdev V, Shizukuda Y, Brenneman CL, Birdsall CW, Waclawiw MA, Arai AE, et al. Left atrial volumetric remodeling is predictive of functional capacity in nonobstructive hypertrophic cardiomyopathy. Am Heart J 2005; 149: 730-736.

15. Moon J, Hong YJ, Kim YJ, Shim CY, Jang Y, Chung N, et al. Extent of late gadolinium enhancement on cardiovascular magnetic resonance imaging and its relation to left ventricular longitudinal functional reserve during exercise in patients with hypertrophic cardiomyopathy. Circ J 2013; 77: 1742-1749.

16. Maron BJ, McKenna WJ, Danielson GK, Kappenberger LJ, Kuhn HJ, Seidman CE, et al. American College of Cardiology/European Society of cardiology clinical expert consensus document on hypertrophic cardiomyopathy: A report of the American College of Cardiology Foundation Task Force on clinical expert consensus documents and the European Society of Cardiology Committee for practice guidelines. J Am Coll Cardiol 2003; 42: 1687-1713.

17. Lang RM, Badano LP, Mor-Avi V, Afilalo J, Armstrong A, Ernande $\mathrm{L}$, et al. Recommendations for cardiac chamber quantification by echocardiography in adults: An update from the American Society of Echocardiography and the European Association of Cardiovascular Imaging. Eur Heart J Cardiovasc Imaging 2015; 16: 233-270.

18. Autore $\mathrm{C}$, Bernabò $\mathrm{P}$, Barillà $\mathrm{CS}$, Bruzzi $\mathrm{P}$, Spirito $\mathrm{P}$. The prognostic importance of left ventricular outflow obstruction in hypertrophic cardiomyopathy varies in relation to the severity of symptoms. $J A m$ Coll Cardiol 2005; 45: 1076-1080.

19. Agostoni P, Bianchi M, Moraschi A, Palermo P, Cattadori G, La Gioia R, et al. Work-rate affects cardiopulmonary exercise test results in heart failure. Eur J Heart Fail 2005; 7: 498-504.

20. Magrì D, Corrà U, Di Lenarda A, Cattadori G, Maruotti A, Iorio A, et al. Cardiovascular mortality and chronotropic incompetence in systolic heart failure: The importance of a reappraisal of current cutoff criteria. Eur J Heart Fail 2014; 16: 201 - 209.

21. Wasserman K, Hansen JE, Sietsema KE, Sue DY. Principles of exercise testing and interpretation. 4th edn. Baltimore: Lippincott Williams \& Wilkins, 2005; 10-65.

22. Corrà U, Mezzani A, Giordano A, Bosimini E, Giannuzzi P. Exercise haemodynamic variables rather than ventilatory efficiency indexes contribute to risk assessment in chronic heart failure patients treated with carvedilol. Eur Heart J 2009; 30: 3000-3006.

23. Nomura A, Konno T, Fujita T, Tanaka Y, Nagata Y, Tsuda T, et al. Fragmented QRS predicts heart failure progression in patients with hypertrophic cardiomyopathy. Circ J 2015; 79: 136-143.

24. Guazzi M, Adams V, Conraads V, Halle M, Mezzani A, Vanhees L, et al; EACPR; AHA. EACPR/AHA Joint Scientific Statement: Clinical recommendations for cardiopulmonary exercise testing data assessment in specific patient populations. Eur Heart J 2012; 33: 2917-2927.

25. Rowin EJ, Maron BJ, Kiernan MS, Casey SA, Feldman DS, Hryniewicz KM, et al. Advanced heart failure with preserved sys- tolic function in nonobstructive hypertrophic cardiomyopathy: Underrecognized subset of candidates for heart transplant. Circ Heart Fail 2014; 7: 967-975.

26. Nistri S, Olivotto I, Betocchi S, Losi MA, Valsecchi G, Pinamonti $\mathrm{B}$, et al. Prognostic significance of left atrial size in patients with hypertrophic cardiomyopathy (from the Italian Registry for Hypertrophic Cardiomyopathy). Am J Cardiol 2006; 98: 960-965.

27. Ciampi Q, Betocchi S, Lombardi R, Manganelli F, Storto G, Losi MA, et al. Hemodynamic determinants of exercise-induced abnormal blood pressure response in hypertrophic cardiomyopathy. $J$ Am Coll Cardiol 2002; 40: 278-284.

28. Kawasaki T, Azuma A, Kuribayashi T, Akakabe Y, Yamano M, Miki S, et al. Vagal enhancement due to subendocardial ischemia as a cause of abnormal blood pressure response in hypertrophic cardiomyopathy. Int J Cardiol 2008; 129: 59-64.

29. Agostoni P, Corrà U, Cattadori G, Veglia F, La Gioia R, Scardovi $\mathrm{AB}$, et al. Metabolic exercise test data combined with cardiac and kidney indexes, the MECKI score: A multiparametric approach to heart failure prognosis. Int J Cardiol 2013; 167: 2710-2718.

30. Imamura T, Kinugawa K, Nitta D, Inaba T, Maki H, Hatano M, et al. Novel scoring system using cardiopulmonary exercise testing predicts prognosis in heart failure patients receiving guideline-directed medical therapy. Circ J 2015; 79: 1068-1075.

31. Guazzi M, Myers J, Arena R. Cardiopulmonary exercise testing in the clinical and prognostic assessment of diastolic heart failure. JAm Coll Cardiol 2005; 46: $1883-1890$.

32. Guazzi M, Labate V, Cahalin LP, Arena R. Cardiopulmonary exercise testing reflects similar pathophysiology and disease severity in heart failure patients with reduced and preserved ejection fraction. Eur J Prev Cardiol 2014; 21: 847-854.

33. Arena R, Myers J, Abella J, Peberdy MA, Bensimhon D, Chase P, et al. Development of a ventilatory classification system in patients with heart failure. Circulation 2007; 115: 2410-2417.

34. Arena R, Owens DS, Arevalo J, Smith K, Mohiddin SA, McAreavey $\mathrm{D}$, et al. Ventilatory efficiency and resting hemodynamics in hypertrophic cardiomyopathy. Med Sci Sports Exerc 2008; 40: 799-805.

35. Olivotto I, Maron MS, Adabag AS, Casey SA, Vargiu D, Link MS, et al. Gender-related differences in the clinical presentation and outcome of hypertrophic cardiomyopathy. J Am Coll Cardiol 2005; 46: $480-487$.

36. Magrì D, De Cecco CN, Piccirillo G, Mastromarino V, Serdoz A, Muscogiuri G, et al. Myocardial repolarization dispersion and late gadolinium enhancement in patients with hypertrophic cardiomyopathy. Circ J 2014; 78: 1216-1223.

37. Briasoulis A, Mallikethi-Reddy S, Palla M, Alesh I, Afonso L. Myocardial fibrosis on cardiac magnetic resonance and cardiac outcomes in hypertrophic cardiomyopathy: A meta-analysis. Heart 2015; 101: $1406-1411$

38. Núñez L, Gimeno-Blanes JR, Rodríguez-García MI, Monserrat L, Zorio E, Coats C, et al. Somatic MYH7, MYBPC3, TPM1, TNNT2 and TNNI3 mutations in sporadic hypertrophic cardiomyopathy. Circ J 2013; 77: 2358-2365.

39. Gómez J, Reguero JR, Morís C, Martín M, Alvarez V, Alonso B, et al. Mutation analysis of the main hypertrophic cardiomyopathy genes using multiplex amplification and semiconductor next-generation sequencing. Circ J 2014; 78: 2963-2971.

40. Hamada M, Shigematsu Y, Ohtani T, Ikeda S. Elevated cardiac enzymes in hypertrophic cardiomyopathy patients with heart failure: A 20-year prospective follow-up study. Circ J 2016; 80: 218-222.

\section{Supplementary Files}

Supplementary File 1

Data S1. Excel calculator for HYPertrophic Exercise-derived Risk HF (HYPERHF) score

Please find supplementary file(s);

http://dx.doi.org/10.1253/circj.CJ-16-0432 